\title{
Fungal keratitis efficient treatments using surface active agents (cetrimide): an overview
}

\begin{abstract}
Early diagnosis of mycotic keratitis in patients is crucial for prompt antifungal therapy. Confirmation of diagnosis in case of mycotic keratitis leads to initiate prompt and accurate therapy that would avoid unnecessary and indiscriminate use of steroids/ antibacterial/antiviral/and antifungal. Immunological methods of mycotic keratitis diagnosis have not been found to be satisfactory, and recent research has been diverted to the use of PCR for the sensitive and early diagnosis at the molecular level. Therapy should be aggressive and most authors advocate dual therapy to avoid the risk of resistance. Typically, the topical antifungal is given every hour initially. We have recommended the use of the surface active agent cetrimide as an effective antimycotic keratitis agent that led to complete inhibition of mycotic ulcers in volunteer cases. Cetrimide showed no pathological effect to eye corneal tissues with no side effects during the treatment course.
\end{abstract}

Keywords: fungal keratitis, mycotic keratitis, surface active agents, antifungal therapy, cetrimide
Volume 2 Issue 3 - 2016

\author{
Yehia AG Mahmoud ${ }^{1,2}$ \\ 'Botany Department, Tanta University, Egypt \\ ${ }^{2}$ Department of Biology, Al-Baha University, Saudi Arabia
}

Correspondence: Yehia AG Mahmoud, Botany Department, Tanta University, Tanta 31527, Egypt, Tel +966 5019949 30, Fax +966 I 7727 |3 8I, Email Yehiamah@gmail.com

Received: May 13, 2016 | Published: July 15, 2016

\section{Introduction}

Keratitis is a term used for description of inflammation of the cornea and the transparent membrane that cover the iris and the pupil of the eye. Various types of eye infections, dry eyes, eyes injury, and a large variety of underlying medical diseases all may lead to keratitis (Figure 1).

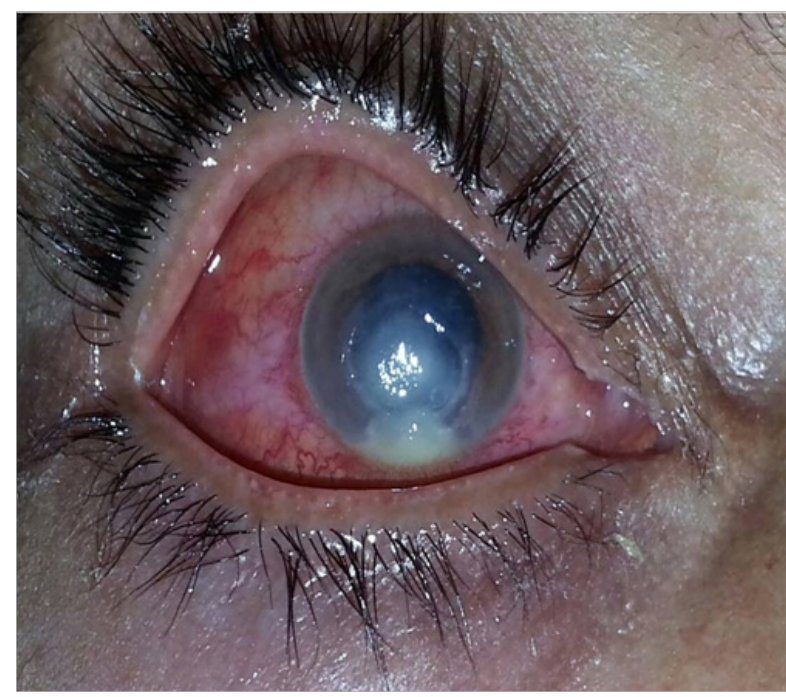

Figure I Typical fungal keratitis: Pyramidal hypopyon, feathery hyphate stromal infiltration, gutter and immune ring.

However, some cases of keratitis result from unknown factors. Generally, more than 70 genera of filamentous fungi and yeast have been identified in fungal keratitis. The predominant causes of fungal keratitis are Aspergillus, Acremonium, Penicillium and Fusarium solani which are all non-pigmented fungi. Although Fusarium and Aspergillus clearly dominate the list, causative agents vary in different geographic areas. Moharram et al., ${ }^{1}$ reported that Fusarium species counted for $13.8 \%$ of fungal keratitis isolated with F. solani being the most common (approximately 10\% of the isolates) in Upper Egypt. However, they have reported a few cases with F. moniliforme and $F$. oxysporum. Fusarium was cultured from $6.8 \% \%^{2}$ to $7.6 \%{ }^{3}$ of cases in delta region in Egypt; $10.7-20 \%$ in India ${ }^{3} 26 \%$ in Thailand; ${ }^{4}$ $11.4-57.2 \%$ microbial keratitis in childhood in the USA; $55.5 \%$ in Argentina $^{6}$ and $62 \%$ in south Florida, USA. ${ }^{7}$ Still, fungal keratitis is the most challenging among corneal ulcers and yet most commonly found in agricultural or developing countries including Malaysia. ${ }^{8-10}$

Most of the literature on corneal ulcer available is from African, Asian and subcontinent. Almost all the reports from India, Nepal, Sri Lanka, and Bangladesh indicate Aspergillus as the most common causal agent of keratomycoses followed by species of Fusarium and Candida. ${ }^{4,11}$ As an exception Rhizopus has been reported as a major cause of mycotic keratitis in Nepal. While in African countries the commonest causal agent is Fusarium $s p$. which followed by Aspergillus and Curvularia sp. However, within the tropics, as many as two third of corneal ulcer may be due to filamentous fungi. ${ }^{12}$ In European countries mycotic keratitis is not common as a mere 3\% of total corneal ulcer patients are reported due to fungi and species of Candida are the commonest isolates ${ }^{4}$ also, Candida albicans is the commonest pathogen of mycotic keratitis in temperate region. ${ }^{13}$ In America Fusarium sp. are the commonest pathogen followed by Candida $s p .{ }^{14}$ According to a World Health Organization report, mortality rate due to keratitis is highest $(0.25$ deaths per 1 million people) in New Zealand. ${ }^{4}$

\section{What are the risk factors for keratitis?}

Major risk factors for the development of keratitis include any break or disruption of the surface layer (epithelium) of the cornea. The use of contact lenses increases the risk for the development of keratitis, especially if when poor hygiene, improper solutions, or over wear are associated with contact-lens use. A decrease in the quality or quantity of tears predisposes the eye to the development of keratitis. ${ }^{2}$ 
The overall outlook of mycotic keratitis is still of great concern. Because of the delay, in patient's presentation to clinicians, in isolation and identification of causative fungus and initiation of antifungal regimen, the vision is very often impaired. In some developing countries corneal ulcer is second commonest cause of blindness after non operated cataract. ${ }^{11}$ Suppurative corneal ulcer may be caused by many factors including bacteria and fungi as discussed earlier. However, Therapeutic contact lens-related bilateral fungal keratitis within the tropics, has been studied by Höflin-Lima \& Roizenblatt. ${ }^{12}$

\section{Recent advancement in diagnostic mycoses}

\section{Immunology based methods}

Serological approaches have been used for many years to established presumptive diagnosis of a number of fungal infections, ${ }^{15-18}$ but the situation is more complicated in case of mycotic keratitis because the cornea is an avascular structure and therefore immunological methods will not be successful.

\section{DNA based methods}

The increasing incidence of fungal infections in immunocompromised individuals has focused attention on the rapid and accurate diagnosis of invasive fungal infections using molecular biological techniques. Nucleic acid hybridization and amplification methods are fundamental to molecular diagnosis. These methods have the potential to provide both high detection rates and identification of specific fungal pathogens. The use of molecular diagnostic tools to detect fungal specific nucleic acid sequences has recently been reviewed, ${ }^{15,19,20}$ and many researchers have reported the usefulness of DNA-based methods for the diagnosis of invasive fungal infections.

We have designed a rapid detection of mycotic keratitis in patients. Ordinary culturing of infection swap is time consuming. SCAR-PCRbased DNA amplification was conducted by using a highly specific primer pairing for detection of fungal chitin synthase gene conserved region, for all samples expected to be infected with fungi; specially by using the primer pairing probability (Forward primer: 5'- TAC AAG CTT ACT ATG TAT AAT GAG GAT -3' + Reverse primer: 5'TTA CTC GAG CTT ATA CTC AAA ATT TTG -3'). The obtained results confirmed that this method gave more accurate, time-saving, as positive visualization of fungal genome was achieved only after 1 day of work, while ordinary cultures consumed 4-14days to visualize the fungal growth. ${ }^{21}$

\section{Suitability, promptness and adaptability of a method}

A diagnostic procedure must be sensitive and accurate for all the fungal genera. A signature molecule of fungi should be identified, which can either be a secretory, cell wall, cytoplasmic molecules, or a conserved region in the chromosome of the fungus. Scraped material from the corneal lesions is very small in amount therefore a method requiring very little amount of patient material will be very useful for diagnostic purpose. Identification based on secretory molecule or protein is not adaptable for these kinds of infections. Only culture based, DNA based or cell wall and cytoplasmic constituent based identification can be used for mycotic keratitis. Further the method should be quick so that an early diagnosis is made and consequently accurate antifungal therapy is initiated instantaneously. ${ }^{4}$

\section{What is the available treatments for keratitis? \\ Antifungal agents}

Treatment of fungal keratitis poses many challenges, at least its poor corneal penetration and limited efficacy of currently available topical antifungal therapies. Actually, there is no one treatment gives prompt satisfactory results in controlling signs and symptoms of keratitis.

Medical treatment of mycotic keratitis is absolutely essential even a delay of a few hours can affect the ultimate visual result. The causative factors must be determined through laboratory analysis of scrapings; medical treatment (i.e., medication) varies depending on the causal agent. Prompt treatment for mycotic keratitis with effective antifungals may prevent loss of vision of the patient. Antibiotics, antifungals, and antivirals have to be used to treat the appropriate organism. Broad-spectrum antibiotics are used immediately, but once the laboratory analysis determines the causative organism the medication may have to be changed. ${ }^{4}$

Sometimes a combination of medication is necessary that depends upon the infection. Antifungal, antibiotic, or antiviral eye drops or ointments are prescribed to cure keratitis, which should be used by patients only under the care of an ophthalmologist. Inappropriate prescriptions or over-the-counter preparations can make symptoms more severe and cause tissue deterioration. Topical corticosteroids can cause great harm to the cornea in patients with herpes simplex keratitis. ${ }^{22}$ The patient with keratitis must be advised to wear a patch to protect the healing eye from bright light, foreign objects, and avoid the lid rubbing against the cornea. The patient should probably consult every day to the ophthalmologist to check on the progress. Although early detection and treatment can cure most forms of keratitis, the infection can cause: glaucoma, permanent scarring, ulceration of the cornea, and blindness. It is extremely important to treat keratitis before corneal tissue is destroyed and scar tissue is formed.

Because the pain is so severe in keratitis, the patient usually welcomes medical attention. However, if the cornea loses its sensitivity (as in trauma, surgery, or damage to the trigeminal nerve), ulcers can develop without accompanying pain. The implications for personal hygiene are evident, especially with children. Hand washing during periods of illness and following toileting is of vital importance as a preventive measure. In today's market, natamycin $5 \%$ is the only commercially available topical agent indicated for the treatment of fungal keratitis, and has been popularly used for filamentous fungi infections. However, there are other therapeutic treatments that include both topical and oral anti-fungal medications. The two most commonly prescribed antifungal treatments are amphotericin $\mathrm{B}$, which is usually used primarily to treat Candida pathogens. In addition, flucytosine can be an alternate treatment, used in conjunction with amphotericin B or miconazole. An eye infected with fungal keratitis before treatment. Notice the extensive neovascularization within the corneal tissue. There are many antifungals available in the market belonging to five major groups: polyenes, imidazoles, triazoles, allylamines, and other include flucytosine and griseofulvin.,

\section{Surface active agents (Cetrimide)}

A new trend in management of fungal infections is to use surface- 
active agents depending upon their safety as a commercial detergents and disinfectant preparation-like povidone iodine, or cholorohexidine and/or cetrimide.

The main problem of commercially available antimycotic keratitis drugs is their instability, poor solubility and penetration ability, restricted pharmacokinetic properties, development of resistant organisms and toxicity even at therapeutic doses. ${ }^{1}$ Therefore, the efficacy of surfactants such as cetrimide compound against human corneal ulcers was evaluated on human volunteers with F. solani. Fusarium solani has been chosen as model because it is nearly, the most prevalent caustic agent all over the world. ${ }^{23}$ Medical treatment of these devastating infections would be aided by the widespread availability of more effective medications. Several investigators concluded that the more the drug penetration and persistence in ocular tissue, the more efficacy and stability of drugs to be used topically in cases of mycotic keratitis. ${ }^{24}$

In our searching for efficient and safe antifungal compounds, we have studied the treatment of mycotic corneal ulcers caused by Aspergillus flavus; using natural, synthetic, and surface active materials. ${ }^{25}$ The survey for antifungal Materials was carried out against Aspergillus flavus which has been isolated from human cases diagnosed to have mycotic ulcers and has been grown on Sabouraud's dextrose. Many natural and synthetic products were chosen to be tested for their inhibitory action against Aspergillus flavus, isolated from different mycotic corneal ulcers. These were apple vinegar, bee propolis, fluconazole, nystatin, a chitosan derivative, an $\mathrm{N}$-heterocyclic bromide derivative, and cetrimide [cationic surfactant $=\mathrm{CH} 3(\mathrm{CH} 2) 15 \mathrm{~N}+(\mathrm{CH} 3) 3 \mathrm{Br}-]$ at $\mathrm{pH} 6.4$ and 5.9 through the initial screening for its antifungal activity. Cut plug method recorded by Pridham et al. ${ }^{25}$ was employed to determine the antifungal activity of the chosen products as follows:

Freshly prepared spore suspension of Aspergillus flavus $(0.5 \mathrm{ml}$ of about 106 spores $/ \mathrm{ml}$ ) was mixed with $9.5 \mathrm{ml}$ of melting sterile Sabouraud's dextrose medium at $45^{\circ} \mathrm{C}$, poured on sterile Petri dishes, and left to solidify at room temperature. Regular wells were made in the inoculated agar plates by a sterile cork borer. Each well was filled with $0.2 \mathrm{ml}$ of each tested compound (either soluble or suspended) to give concentration of $20 \mathrm{mg} / \mathrm{ml}$. All plates were incubated at $27^{\circ} \mathrm{C}$ for 3days. Then diameters of inhibition zones were recorded, and compared for all plates.

The activity (fungus inhibition zone) of the most tested materials (Chitosan derivatives, N-heterocyclic-bromide derivative, Apple vinegar, Bee propolis, Nystatin, Fluconazole and Cetrimide has proved that cetrimide has the most observable inhibition activity among all the tested materials (Table 1).

Accordingly, several parameters were measured to ensure the promising antifungal activity of cetrimide, and safety to be used in treatment of mycotic corneal ulcers, also nystatin was studied for comparison (a well-known anti-fungal agent as a positive control); untreated fungal growth was taken as a negative control (at 0.0 concentration of chosen materials). Study has been conducted at the minimal inhibitory concentration (MIC) for Aspergillus flavus with cetrimide which was sharply defined to be of $0.01 \mathrm{mg} / \mathrm{mL}$ that gave a complete inhibitory effect on the fungus. However, nystatin needs higher concentration to be noticeably effective even until $1 \mathrm{mg} / \mathrm{ml}$.

Improvement tests for cetrimide safety to be used in topical treatment for human mycotic keratitis were achieved by histopathological studies on healthy rabbit corneas. In comparison with control, cetrimide-treated corneal section possessed no significant toxic effects; the corneal tissues appeared with normal compact epithelium, and endothelium, regularly arranged stromal lamellae, and no vacuolation in Descemet's endothelial complex. On the other hand, nystatin-treated cornea possessed increased endothelial thickness, epithelial desquamation, and severe stromal edema with vacuolation due to infiltration of aqueous. ${ }^{25}$

Current successful data for cetrimide efficacy against F. solani was confirmed by the very low surviving cell number in the presence of different cetrimide concentration. ${ }^{23}$ The healing of human corneal ulcer was surprising with cetrimide as a simply prepared solution in distilled water with very low concentration where the MIC value of cetrimide and nystatin was similar and concluded to be $(100 \mu \mathrm{g} . \mathrm{mL}-$ 1). ${ }^{23}$

Examination of corneal sections treated with cetrimide indicated no significant toxic effects and appeared with normal corneal tissues of experimental animals. Mirza \& Hart \& Dong et al., ${ }^{26,27}$ have been successively treated a (young man) patient with ketoconazole and miconazole, but these compounds have a high price and the patient might develop resistant to it after a prolonged period of time treatments. Usage of bee propolis for healing of mycotic keratitis was investigated. Orsi et al., ${ }^{28}$ have mentioned that propolis has been used in folk medicine since ancient times that might be due to its safe biological properties, such as antimicrobial, anti-inflammatory, antioxidant and immunomodulatory activities. Our obtained results indicated that cetrimide alone has a high capacity and short treatment time course in order to heal fungal keratitis caused by F. solani in vivo. ${ }^{23}$ Cetrimide with other components-like bee propolis, and fluconazole gave a good healing outcome, but take a longer time about 6 weeks to achieve a good recovery as illustrated in (Figure 2). ${ }^{23}$

Table I General survey for inhibition of Aspergillus flavus growth (inhibition zone diameter, $\mathrm{mm}$ ) by tested materials. ${ }^{25}$

\begin{tabular}{|c|c|c|c|c|c|c|c|}
\hline $\begin{array}{l}\text { Antifungal } \\
\text { agents }\end{array}$ & $\begin{array}{l}\text { Chitosan } \\
\text { derivative }\end{array}$ & $\begin{array}{l}\text { N-heterocyclic-bromide } \\
\text { derivative }\end{array}$ & $\begin{array}{l}\text { Apple } \\
\text { vinegar }\end{array}$ & $\begin{array}{l}\text { Bee } \\
\text { propolis }\end{array}$ & Nystatin & Fluconazole & Cetrimide \\
\hline Inhibition zone & $0.9 \pm 0.08$ & $1.4 \pm 0.1$ & $1.1 \pm 0.1$ & $1.2 \pm 0.11$ & $2.8 \pm 0.20$ & $2.7 \pm 0.2$ & $5.7 \pm 0.6$ \\
\hline
\end{tabular}




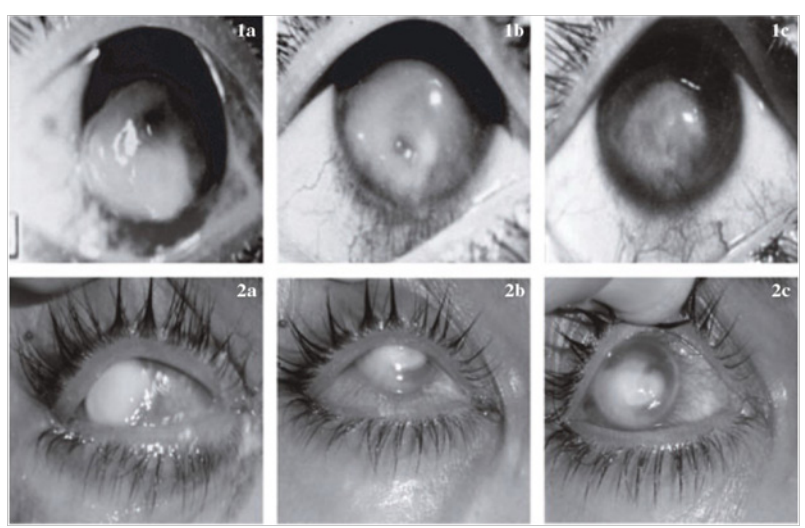

Figure 2 Corneal mycotic ulcers with Fusarium solani as a causative agent (la case and $2 \mathrm{a}$ other case) treated with: (i) application of three drops (approximately $0.3 \mathrm{~mL})$ of cetrimide $(10 \mathrm{mg} \cdot \mathrm{mL}-\mathrm{I})$ three times daily for $3(\mathrm{Ib})$ and 6weeks ( $\mathrm{Ic})$; (ii) application of three drops $(0.3 \mathrm{~mL})$ of mixture containing $10 \mathrm{mg} \cdot \mathrm{mL}-\mathrm{I}$ ) of cetrimide, $4 \mathrm{mg} \cdot \mathrm{mL}-\mathrm{I}$ ) of bee propolis in honey and $2 \mathrm{mg} \cdot \mathrm{mL}-\mathrm{I}$ ) of fluconazole in distilled water (mixture, $\mathrm{pH}$ equal to 5.7), three times daily for $3(2 b)$ and 6weeks (2c).

\section{Conclusion}

Therefore, from this study, it could be concluded that cetrimide is a good antimycotic compound that could inhibit mycotic ulcers efficiently. Cetrimide showed no pathological effect to eye corneal tissues with no side effects during the treatment course. Approximately one third of fungal infections fail to respond to medical treatment and may result in corneal perforation. In these cases, a therapeutic penetrating keratoplasty is necessary. Penetrating keratoplasty generally should be performed within 4weeks of presentation. A small number of patients have been treated successfully with a conjunctival flap. The main goals of surgery are to control the infection and to maintain the integrity of the globe. Topical antifungal therapy, in addition to systemic fluconazole or ketoconazole, should be continued following penetrating keratoplasty. The use of topical corticosteroids in the postoperative period remains controversial.

To sum up the necessity of a rapid diagnostic method is required. This necessity has prompted the development of a test and other tools that may lead to prompt initiation of antifungal therapy and good prognosis of the infection.

\section{Authors' contributions}

YM designed the research and methodology; collecting the data and compiled all the data and literature; revised the review and approved it for submission; responsible for the future questions from readers as the corresponding authors.

\section{Acknowledgements}

The author expresses his sincere thanks for prof. Sameh Mohamed El-Shourbagy of Ophthalmology Department, Faculty of Medicine, Tanta University, Tanta, Egypt for his continuous help and encouragement for looking up for an effective and safe treatment of Mycotic keratitis.

\section{Conflict of interest}

The author declares no conflict of interest.

\section{References}

1. Moharram AM, Kader MIAA, Al Hussaini AK, et al. Studies on mycotic keratitis in Assiut governorate. In: Proceedings of the $2^{\text {nd }}$ International Conference on Fungi: Hopes \& Challenges, Cairo, Egypt; 1999. 1:133146.

2. Mahmoud YAG, Abu El Souod SM, El Shourbagy MS, et al. Study on human corneal ulcers in Tanta University Ophthalmology hospitals with special attention to mycotic keratitis. Bull Fac Sci Assuit Univ. 2004;33:1-15.

3. Arora R, Venkateswarlu K, Mahajan VM. Keratomycosis a retrospective histopathologic and microbiologic analysis. Ann Ophthalmol. 1988;20(8):306-310.

4. Imwidthaya P. Mycotic keratitis in Thailand. $J$ Med Vet Mycol. 1995;33(1):81-82.

5. Cruz OA, Sabir SM, Capo H, et al. Microbial keratitis in childhood. Ophthalmology. 1993;100(2):192-196.

6. Albrecht MC, Gomez LA. Opportunistic fungi isolated from ocular lesions. Rev Argent Micol. 1993;16:11-14.

7. Rosa RJ, Miller D, Alfonso EC. The changing spectrum of fungal keratitis in south Florida. Ophthalmology. 1994;101(6):1005-1013.

8. Norina TJ, Raihan S, Bakiah S, et al. Microbial keratitis: etiological diagnosis and clinical features in patients admitted to Hospital University Sains Malaysia. Singapore Med J. 2008;49(1):67-71.

9. Kursiah MR, Sharif FM, Balaravi P. Retrospective review of corneal ulcers in Ipoh Hospital. Med J Malaysia. 2008;63(5):391-394.

10. Hooi SH, Hooi ST. Culture-proven bacterial keratitis in a Malaysian general hospital. Med J Malaysia. 2005;60(5):614-623.

11. Hemo I, Peer J, Polacheck I. Fusarium oxysporum keratitis. Ophthalmologica. 1989;198(1):3-7.

12. Höflin Lima AL, Roizenblatt R. Therapeutic contact lens-related bilateral fungal keratitis. CLAO J. 2002;28(3):149-150.

13. Laverde S, Moncada LH, Restrepo A, et al. Mycotic keratitis; 5 cases caused by unusual fungi. Sabouraudia. 1973;11(2):119-123.

14. Luque AG, Nanni R, De Bracalenti BJ. Mycotic keratitis caused by Curvularia lunata var. aeria. Mycopathologia. 1986;93(1):9-12.

15. Gugani HC, Talwar RS, Njoku Obi AN, et al. Mycotic keratitis in Nigeria. A study of 21 cases. Br J Ophthalmol. 1976;60(9):607-613.

16. Hagan M, Wright E, Newman M, et al. Causes of suppurative keratitis in Ghana. Br J Ophthalmol. 1995;79(11):1024-1028.

17. Mselle J. Fungal keratitis as an indicator of HIV infection in Africa. Trop Doct. 1999;29(3):133-135.

18. Ormerod D. Causation and management of microbial keratitis in subtropical Africa. Ophthalmolog. 1987;94(12):1662-1668.

19. Coster DJ, Wilhelmus KR, Michaud R, et al. A comparison of acyclovir and idoxuridine as treatment for ulcerative herpetic keratitis. $\mathrm{Br} J$ Ophthalmol. 1980;64(10):763-765.

20. Harris DJ, Stulting RD, Waring GO, et al. Late bacterial and fungal keratitis after corneal transplantation. Spectrum of pathogens, graft survival, and visual prognosis. Ophthalmology. 1980;95(10):1450-1457.

21. Abu El Souod SM, Mahmoud YAG, El Shourbagy SM, et al. Simple, fast, and accurate method for detection of human mycotic keratitis. Egypt $J$ Bot. 2013:439-451.

22. Ahmad S, Khan Z, Mustafa AS, et al. Seminested PCR for diagnosis of 
Candidemia: Comparison with culture, antigen detection, and biochemical methods for species identification. J Clin Microbiol. 2002;40(7):24832489 .

23. Mahmoud YA. In vitro and in vivo antifungal activity of cetrimide (cetyltrimethyl ammonium bromide) against fungal keratitis caused by Fusarium solani. Mycoses. 2007;50(1):64-70.

24. Gopinathan U, Garg P, Fernandes M, et al. The epidemiological features and laboratory results of fungal keratitis: a 10-year review at a referral eye care center in South India. Cornea. 2002;21(6):555-559.

25. Mahmoud YA, Abu El Souod SM, El Shourbagy SM, et al. Treatment of Aspergillus flavus mycotic ulcers, using natural. Synthetic, and surface active materials. Journal of King Abdulaziz University Bulletin "Science". 2011;23(1):117-133.
26. Kanungo R, Srinivasan R, Rao RS. Acridine orange staining in early diagnosis of mycotic keratitis. Acta Ophthalmol (Copenh). 1991;69(6):750-753.

27. Sharma S, Kunimoto DY, Gopinathan U, et al. Evaluation of corneal scraping smear examination methods in the diagnosis of bacterial and fungal keratitis:A survey of eight years of laboratory experience. Cornea. 2002;21(1):643-647.

28. Sharma S, Silverberg M, Mehta P, et al. Early diagnosis of mycotic keratitis: predictive value of potassium hydroxide preparation. Indian $J$ Ophthalmol. 1998;46(1):31-35. 